Research Paper

\title{
Improving stereotactic radiotherapy (SRT) planning process for brain metastases by Cyberknife system: reducing dose distribution in healthy tissues
}

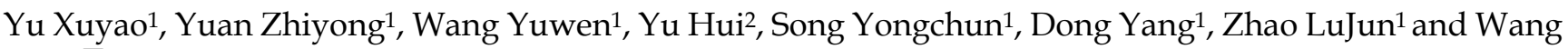 \\ Ping ${ }^{1 \times}$ \\ 1. Department of Radiotherapy, Tianjin Tumour Hospital, Tianjin, China \\ 2. Biomedical Engineering, Tianjin University, Tianjin, China \\ $\triangle$ Corresponding author: Wang Ping, Department of Radiotherapy, Tianjin Tumour Hospital, Huanhu Middle Road, Hexi District, Tianjin, 300060 , China. \\ Telephone:086-022-2334-1405, Email: wangping@tjmuch.com
}

(c) The author(s). This is an open access article distributed under the terms of the Creative Commons Attribution License (https://creativecommons.org/licenses/by/4.0/). See http://ivyspring.com/terms for full terms and conditions.

Received: 2019.10.11; Accepted: 2020.03.29; Published: 2020.04.13

\begin{abstract}
Purpose: To pursue high precision dose in lesions and steeper dose fall-off in healthy tissues of brain metastases stereotactic radiotherapy (SRT), this study investigated an opitimized planning by comparison different prescription dose line in the treatment of brain metastases using Cyberknife (CK) Robotic Radiosurgery System.

Methods: 77 patients (92 lesions) brain metastases patients CK SRT plans were replanned with $50 \%-80 \%$ (5\% internal) prescription dose line to cover more than $95 \%$ of the planned target volume (PTV), under the same collimator by Multiplan System. Under the precondition of guaranteeing plans all meet the clinical requirements, the plan evaluation paraments (conformal index $(\mathrm{Cl})$ and homogeneity index $(H I)$ ), plan treatment time parameters (the total number of beams and monitor units (MU)) and dose distribution of organs at risk (OAR) and healthy brain tissues adjacent to the PTV were analyzed respectively.

Resluts: Compared with $70 \%$ plans, $65 \%$ plans had: 1$)$ average dose $\left(D_{\text {mean }}\right)$ and maximum dose $\left(D_{\max }\right)$ of healthy brain tissue outside of the PTV reduced $11.83 \%$ and $5.97 \%$ markedly; 2) $D_{\text {mean }}$ and $D_{\max }$ of brainstem decreased $11.43 \%$ and $2.86 \%$; 3 ) the volumes of whole brain minus the tumors received a single dose equivalence of $12 \mathrm{~Gy} / 14 \mathrm{~Gy}(\mathrm{~V} 12 \mathrm{~Gy} / \mathrm{V} 14 \mathrm{~Gy})$ had marked decline. The dose fall-off was considerably faster in the $60 \%-65 \%$ plans around the PTV and the maximum dose of healthy tissue was prominently lower. While the difference in $\mathrm{Cl}$ and $\mathrm{HI}$ between different plans was not obvious, the plan treatment time was a little higher in $60 \%-65 \%$ plans than $70 \%-80 \%$ plans.

Conclusions: Choosing a relatively lower isodose as the prescription dose line for brain metastases CK SRT planing could improve the dosimetry index of target and immensely reduce high dose in healthy brain tissue and OAR.
\end{abstract}

Key words: CyberKnife, stereotactic radiotherapy, brain metastases, treatment plan, prescription dose line

\section{Introduction}

With improvements in control of systemic disease, better radiologic detection, and prolonged survival, the brain metastases in patients with cancer are more frequent than primary brain tumors [1]. Brain metastasis occurs in approximately $20-40 \%$ of patients with cancer, and that has become an important factor affecting the survival of patients [2]. Numerous studies have shown stereotactic radiotherapy (SRT) has better local control (LC) of the treated brain metastases than surgery, and solves the shortcomings of whole brain radiotherapy (WBRT) including low dose distribution in the tumor area and 
high side effects. SRT has been known as the best available treatment for cancer patients with one to three brain metastases [3].

CyberKnife (CK) SRT system uses real-time X-ray exposure to locate the patients' head, and the linear accelerator is controlled by the six-dimensional robotic arm for precise positioning radiotherapy according to the results of the tracking algorithm. That can effectively improve SRT treatment accuracy and reduce the damage to healthy tissues $[4,5,6]$. Therefore, CK SRT has been widely used for 1-3 brain metastases patients clinical treatment.

Park S $\mathrm{H}$ et al. [7] used Gamma knife radiosurgery for multiple brain metastases with mean prescription isodose at the tumor margin was $50 \%$ (range: $40 \%-60 \%$ ). Sio $\mathrm{T} \mathrm{T}$ et al. [8] adopted the $80 \%$ isodose line for the prescribed dose in the CK SRS plans for patients with brain metastases. Takashi U et al. [9] selected $59 \%-79 \%$ prescribed isodose line for head tumors CK planning. Zindler J D et al. [10] studied stereotactic radiosurgery for 4-10 brain metastases and considered dose inhomogeneity, with a maximum allowed dose within the planning target volume (PTV) of $140 \%$ prescribed dose $(70 \%$ prescription isodose).

Isodose line from $50 \%$ to $80 \%$ isodose is often chosen as the prescribed dose line for brain metastases CK SRT planning in clinical practice. There is very little related researches about the effect of prescription dose line on dosimetry distribution in healthy brain tissues during CK SRT planning in detail, and which is not any uniform standard.

Several studies show that higher total irradiation dose, fraction dose and irradiated volume of healthy brain tissue adjacent to lesions are associated with more clinically meaningful cases of cerebral radiation necrosis (CRN) [11]. During CK SRT planning, choosing different isodose line of global maximum as the prescribed dose curve will not only vary the maximum dose values in the tumor area, and cause different dose distribution of organs at risk (OAR) and healthy brain tissue. However, previous studies have shown that CRN is related to dose distribution in healthy brain tissue around the PTV and V12/V14 (surrounding brain volume circumscribed with a single dose equivalence of 12/14 Gy.

Therefore, in this paper, we conducted a retrospective study to analyzed dosimetry distribution of healthy tissues in CK SRT plans for brain metastases with difference prescription dose line, $50 \%-80 \%$ (5\% internal) isodose, and the purpose of it was to identify the optimal prescription isodose line for improving CK treatment: 1) to deliver large dose to the tumor, 2) achieve minimizes the amount of radiation delivered the healthy tissues.

\section{Material and Methods}

\section{Patient characteristics}

From January 2016 to June 2019, 77 patients were treated with image guided SRT using CK system (CyberKnife III, Accuray Inc., Sunnyvale, CA) for brain metastasis) at Tianjin Medical University Cancer Institute and Hospital. All patients participating in this study had not metastases to other oragans and not undergone pre- CK SRS metastasectomy or pre- or concurrent-to- CK SRS WBRT. Twenty-three patients $(29.9 \%)$ had a KPS of $<70$ and all patients were in RTOG-RPA class 2 or 3 . This report analyzed a head-to-head, quantitative comparison of dosimetry profiles between the different treatment plans for 92 brain metastases lesions (15 patients with two brain metastatic lesions (included 30 lesions)) by Multiplan system (Accuray, Sunnyvale, CA, USA) software program, which determines the lesions volume based on computed tomography (CT) and enhanced T1-weighted magnetic resonance imaging (MRI) fusion. Table 1 shows the patient characteristics.

Table 1. Clinical characteristics of 77 patients.

\begin{tabular}{ll}
\hline Median age (years)(range) & $53(27-81)$ \\
Age $\geq 60$ & 28 \\
Age $<60$ & 49 \\
Sex & \\
Male & 32 \\
Female & 45 \\
Primary cancer & \\
Liver & 15 \\
Breast & 20 \\
Lung & 45 \\
Median KPS score & $72(51-100)$ \\
KPS $\geq 70$ & 54 \\
KPS $<70$ & 23 \\
Multiple vs. Single & \\
Multiple metastases & 15 \\
Single metastasis & 62 \\
Location of tumor & \\
Cerebral hemisphere & 47 \\
Cerebellum & 35 \\
Brainstem & 10 \\
Tumor volume (cm 3$)$ (range) & $4.6(0.05-9.89)$ \\
$5.0-10.0$ & 17 \\
$1.0-5.0$ & 64 \\
$<1.0$ & 11 \\
Prescription dose(Gy)(range) & $12-32(24)$ \\
Fraction(range) & $1-4(3)$ \\
\hline
\end{tabular}

\section{Target delineation and CK SRT treatment planning}

Overall skull CT scan for the patients was performed by Philips Brilliance Big Bore CT (16 rows), with the thickness of scan layer of $1.5 \mathrm{~mm}$. T1-weighted magnetic resonance imaging scan with Siemens 1.5 was registered to CT. CT and MRI images were used for the delineation of the gross tumor 
volume (GTV) and the critical organ structures (also called OAR) including brainstem, eyes, lens, optic nerve, optic chiasm, and pituitary gland. The targeting error of brain CK SRT under skull tracking is $0.956 \mathrm{~mm}$, a $1.6-\mathrm{mm}$ margin was added to the GTV to create the plan tumor volume (PTV), expects the patients with brainstem metastasis [12].

Five dose-limiting shells $(2 \mathrm{~mm}, 3 \mathrm{~mm}, 5 \mathrm{~mm}, 7$ $\mathrm{mm}, 9 \mathrm{~mm}$ ) away from the each PTVs were created to optimize the dose distribution to healthy brain tissues. Based on the delineation results and requirements, the planner conducted the design and optimization of the CK SRT plans by adopting the reverse optimization and nonisocentric algorithm through the MultiPlan system. A high-resolution calculation step was performed in the evaluation step to finalize the CK SRT plans. For each patient, seven different CK SRT plans were designed by using different prescription isodose ranged from $50 \%$ to 80\% (including Plan_50\%, Plan_55\%, Plan_60\%, Plan_65\%, Plan_70\%, Plan_75\%, Plan_80\%). The prescription isodose must cover more than $95 \%$ of the PTV volume in all plans. All of the plans for the same patient followed the same dose limit conditions of OAR, without the iris or MLC system, in order to ensure the consistency of beam data in plans. During CK SRT Plannging for 15 patients with two brain metastatic lesions (included 30 lesions), the prescription dose line of each lesions plans should be consistent. And high-resolution calculation step was performed in the evaluation step to finalize CK SRT plans.

\section{Evaluation of CK SRT plans}

Firstly, the minimum dose $\left(D_{\text {min }}\right)$, maximum dose $\left(D_{\max }\right)$ and mean dose $\left(D_{\text {mean }}\right)$ were evaluated and compared in the PTV, OAR and healthy brain tissue around the PTV (PTV+2 meant 2-mm-thick healthy brain tissue adjacent to the PTV, PTV+6 meant 4-mm-thick healthy brain tissue adjacent to the PTV+2). Secondly, the volume of whole brain tissue minus the PTV received 12 Gy/14 Gy (V12Gy/ V14Gy) in a single fraction was calculated by the LQ model. Finally, the plan evaluation paraments (conformal index $(C I)$ and homogeneity index $(H I)$ ) and the plan treatment time parameters (the total number of beams and monitor units $(M U)$ ) were compared.

CI was commonly used to evaluate CK SRT plans [13] and calculated as follows:

$$
C I=P I V / T I V
$$

Where PIV was the volume included by prescription isodose, and TIV was the tumour volume covered by prescription isodose volume. This definition of $C I$ is different than the radiation therapy oncology group (RTOG) definition, which is PIV divided by total tumor volume [14]. The closer the value of $C I$ is 1 , the better the plan.

\section{Results}

The results of different CK SRT plans using 50\% and $80 \%$ prescription isodose for two patients (Patient 1 with metastases in the right lateral ventricle, Patient 2 with brainstem metastases) were shown in Figure 1.
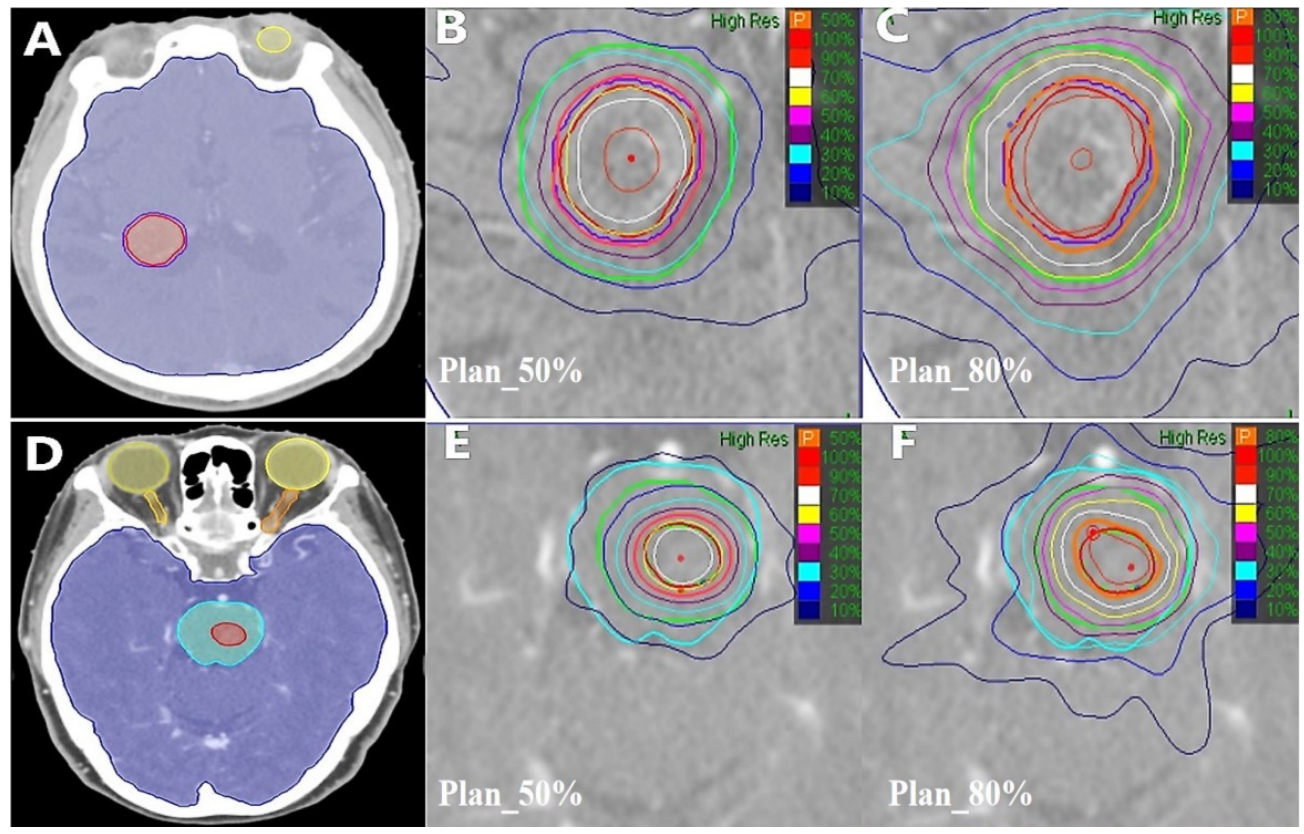

Figure 1. Different CK SRT plans for brain metastases patients. The representative patient had axial images taken, (A) and (D) were Patient 1 with metastases in the right lateral ventricle and Patient 2 with brainstem metastases. The red and purple lines area indicate the GTV and the PTV, respectively. Blue, orange and yellow lines represent Brainstem, Optic Nerves, and Eyes, respectively. (B) and (E) represent 50\% prescription isodose covered more than $95 \%$ of the Patient 1 PTV and Patient 2 GTV (Plan_50\%). (C) and (F) represent $80 \%$ prescription isodose covered more than $95 \%$ of the Patient 1 PTV or Patient 2 GTV (Plan_ $80 \%$ ).Green line zones were covered 6-mm thick healthy brain tissue adjacent to the PTV or the GTV. 


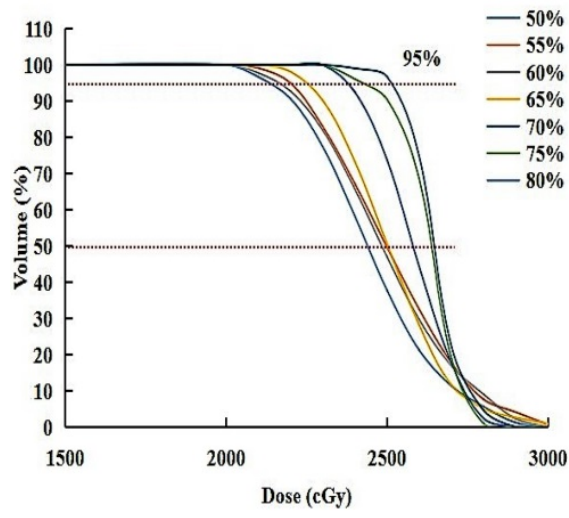

(A)

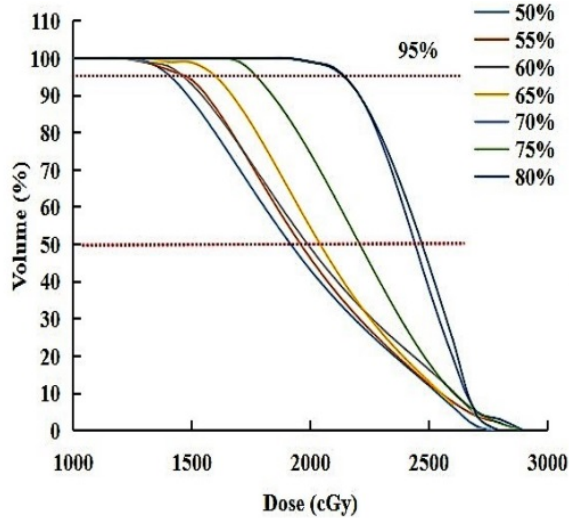

(B)

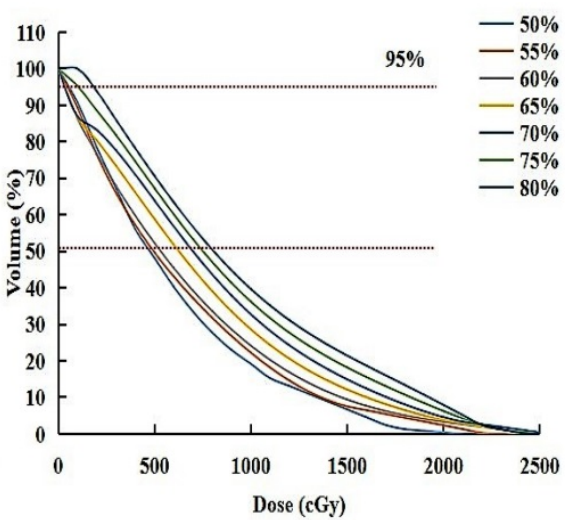

(C)

Figure 2. PTV+2, PTV+6 and brainstem dose volume histogram (DVH) of patient 2 with brainstem metastases. (A) and (B) were dosimetry distribution of 2-mm-thick and 6-mm-thick healthy brain tissue adjacent to the PTV. (C) was the dosimetry distribution of Brainstem.

Table 2. The dose distribution of healthy brain tissue around the PTV (using percent of the global maximum dose).

\begin{tabular}{|c|c|c|c|c|c|c|}
\hline & \multicolumn{2}{|l|}{ PTV } & \multicolumn{2}{|l|}{ PTV+2 } & \multicolumn{2}{|l|}{ PTV+6 } \\
\hline & $D_{\text {mean }}$ & $D_{\min }$ & $D_{\max }$ & $D_{\text {mean }}$ & $D_{\max }$ & $D_{\text {mean }}$ \\
\hline Plan_50\% & $63.62 \% \pm 3.80 \%$ & $53.51 \% \pm 1.46 \%$ & $57.28 \% \pm 3.20 \%$ & $47.57 \% \pm 2.56 \%$ & $53.40 \% \pm 3.11 \%$ & $35.86 \% \pm 2.03 \%$ \\
\hline Plan_55\% & $65.13 \% \pm 2.71 \%$ & $54.61 \% \pm 1.96 \%$ & $58.02 \% \pm 4.01 \%$ & $48.50 \% \pm 2.17 \%$ & $56.12 \% \pm 3.49 \%$ & $36.24 \% \pm 1.96 \%$ \\
\hline Plan_60\% & $67.34 \% \pm 2.92 \%$ & $55.08 \% \pm 1.73 \%$ & $60.25 \% \pm 3.14 \%$ & $50.09 \% \pm 2.87 \%$ & $58.84 \% \pm 2.99 \%$ & $39.8 \% \pm 3.23 \%$ \\
\hline Plan_65\% & $68.26 \% \pm 3.16 \%$ & $56.12 \% \pm 2.04 \%$ & $65.45 \% \pm 3.28 \%$ & $51.27 \% \pm 3.58 \%$ & $63.35 \% \pm 3.50 \%$ & $39.96 \% \pm 3.67 \%$ \\
\hline Plan_70\% & $70.82 \% \pm 2.75 \%$ & $62.39 \% \pm 1.80 \%$ & $69.63 \% \pm 3.77 \%$ & $54.47 \% \pm 4.01 \%$ & $67.37 \% \pm 3.37 \%$ & $45.32 \% \pm 2.15 \%$ \\
\hline Plan_75\% & $71.94 \% \pm 3.03 \%$ & $63.61 \% \pm 1.54 \%$ & $70.82 \% \pm 3.65 \%$ & $57.12 \% \pm 3.44 \%$ & $69.34 \% \pm 2.86 \%$ & $53.42 \% \pm 4.21 \%$ \\
\hline Plan_80\% & $76.56 \% \pm 3.17 \%$ & $69.10 \% \pm 1.74 \%$ & $72.49 \% \pm 3.09 \%$ & $62.06 \% \pm 3.97 \%$ & $70.88 \% \pm 3.24 \%$ & $57.35 \% \pm 2.65 \%$ \\
\hline
\end{tabular}

*PTV+2 meant 2-mm-thick healthy brain tissue adjacent to the PTV, PTV+6 meant 4-mm-thick healthy brain tissue adjacent to the PTV+2

These results illustrated that 1) the radiation around PTV was more divergent with the value of the prescription dose line increased from $50 \%$ to $80 \%$. For example, the $30 \%$ isodose (blue line) was included in the PTV+6 area in Plan_50\% (as shown in Figure.1(B) and (E)), but not in Plan_80\% (as shown in Figure.1(C) and(F)). 2) OAR (notably, the brainstem) and healthy brain tissue were characterized as less irradiated areas and had a closed isodose in Plan_50\%. These illustrated that using lower prescription isodose could significantly reduce dose distribution in plans.

In order to quantify the difference in dose distribution, PTV $+2, \mathrm{PTV}+6$ and brainstem dose volume histogram $(\mathrm{DVH})$ of patient 2 were compared in Figure 2. Although Plan_80\% had the maximum slope of DVH, the dose covering more than $50 \%$ and $95 \%$ volume of the PTV+2 and PTV+6 area was the biggest in it, especially. $D_{\text {mean }}$ was kept in a lower range in Plan_50\% to Plan_65\%. The same result was found in the brainstem region, especially the dose covering more than 50\% volumes of it. These demonstrated that more healthy brain tissue and OAR adjacent tumor tissue received higher dose in CK SRT plans with higher prescription dose line.

\section{Statistical analysis of dosimetric distribution}

The dosimetric distribution in CK SRT plans for 92 intracranial tumours were shown in Table 2. The value of $D_{\min }, D_{\max }$ and $D_{\text {mean }}$ were expressed as percent of the global maximum dose in plans. The $D_{\text {min }}$ and $D_{\text {mean }}$ of the PTV were slightly higher with increasing value of prescription isodose from $50 \%$ to $80 \%$. In Plan $65 \%, D_{\text {mean }}$ and $D_{\max }$ of the PTV+6 were significantly reduced by $11.83 \%$ and $5.97 \%$, compared with Plan_70\%. These results further confirmed that the dosimetric distribution exhibited better convergence and healthy brain tissues around the PTV received a lower radiation dose in Plan_65\%.

The dosimetry distribution of OAR was shown in Table 3. The Dmax and Dmean of the optic chiasm and optic nerve were lower in plans with lower isodose as the prescription dose curve. Compared with Plan_70\%, the Dmean and Dmax dose of the brainstem were reduced $11.43 \%$ and $2.86 \%$ respectively in Plan_60\% for patients with brainstem metastasis. The brain metastasis selected in this study was located far from the eyes, and the eyes (especially lens) had the highest protection priority during the CK SRT plan designing. Thus, the dosimetry distribution of the lens was the lowest and not significantly different between plans.

The PTV Coverage, HI, MU and CI of different CK SRT plans for 77 patients were statistically analyzed, and the results were shown in Table 4. PTV Coverage of different CK SRT plans was similar. It indicated that the PTV dose cloud meet the requirement of the clinical dose prescribed in each plan. 
Table 3. The dosimetry distribution of OAR (using percent of the global maximum dose).

\begin{tabular}{|c|c|c|c|c|c|c|}
\hline & \multicolumn{2}{|l|}{ Brainstem } & \multicolumn{2}{|l|}{ Optic Nerves } & \multicolumn{2}{|l|}{ Optic Chiasm } \\
\hline & $D_{\max }$ & $D_{m e a n}$ & $D_{\max }$ & $D_{m e a n}$ & $D_{\max }$ & $D_{\text {mean }}$ \\
\hline Plan_50\% & $63.62 \% \pm 3.17 \%$ & $20.75 \% \pm 1.55 \%$ & $14.84 \% \pm 4.17 \%$ & $7.51 \% \pm 3.76 \%$ & $18.84 \% \pm 4.07 \%$ & $5.39 \% \pm 1.17 \%$ \\
\hline Plan_55\% & $65.13 \% \pm 2.44 \%$ & $21.20 \% \pm 1.34 \%$ & $15.05 \% \pm 2.98 \%$ & $7.59 \% \pm 2.91 \%$ & $19.25 \% \pm 4.30 \%$ & $5.44 \% \pm 1.49 \%$ \\
\hline Plan_60\% & $67.34 \% \pm 3.09 \%$ & $22.13 \% \pm 1.46 \%$ & $16.12 \% \pm 3.70 \%$ & $7.62 \% \pm 2.95 \%$ & $20.43 \% \pm 4.12 \%$ & $5.80 \% \pm 1.96 \%$ \\
\hline Plan_65\% & $68.26 \% \pm 2.91 \%$ & $22.40 \% \pm 1.19 \%$ & $16.45 \% \pm 3.24 \%$ & $8.03 \% \pm 3.12 \%$ & $22.17 \% \pm 3.37 \%$ & $6.11 \% \pm 2.31 \%$ \\
\hline Plan_70\% & $70.82 \% \pm 2.84 \%$ & $25.29 \% \pm 2.07 \%$ & $19.05 \% \pm 2.78 \%$ & $8.23 \% \pm 3.51 \%$ & $23.75 \% \pm 3.37 \%$ & $7.28 \% \pm 2.15 \%$ \\
\hline Plan_75\% & $71.94 \% \pm 3.15 \%$ & $29.02 \% \pm 2.13 \%$ & $20.46 \% \pm 3.14 \%$ & $9.25 \% \pm 3.18 \%$ & $24.73 \% \pm 2.16 \%$ & $7.42 \% \pm 1.98 \%$ \\
\hline Plan_80\% & $76.56 \% \pm 2.97 \%$ & $31.25 \% \pm 1.83 \%$ & $22.49 \% \pm 3.09 \%$ & $10.06 \% \pm 3.62 \%$ & $25.88 \% \pm 3.24 \%$ & $7.51 \% \pm 2.14 \%$ \\
\hline
\end{tabular}

*Brainstem dose statistics were from 10 patients(10 lesions) with brainstem metastasis. Optic nerves were superposition of both sides.

Table 4. The statistical result of index for different plans

\begin{tabular}{|c|c|c|c|c|c|}
\hline & PTV Coverage & Conformal index $(C I)$ & Homogeneity index (HI) & Total Beam counts & Machine hop(MU) \\
\hline Plan_50\% & $96.02 \% \pm 0.81 \%$ & $1.20 \pm 0.10$ & $1.41 \pm 0.37$ & $108 \pm 11$ & $7276 \pm 256$ \\
\hline Plan_55\% & $95.83 \% \pm 0.52 \%$ & $1.20 \pm 0.09$ & $1.40 \pm 0.39$ & $102 \pm 8$ & $7154 \pm 217$ \\
\hline Plan_60\% & $96.00 \% \pm 0.74 \%$ & $1.15 \pm 0.07$ & $1.36 \pm 0.21$ & $105 \pm 14$ & $6990 \pm 187$ \\
\hline Plan_65\% & $95.98 \% \pm 0.36 \%$ & $1.16 \pm 0.09$ & $1.36 \pm 0.30$ & $110 \pm 12$ & $6951 \pm 135$ \\
\hline Plan_70\% & $96.12 \% \pm 0.29 \%$ & $1.14 \pm 0.08$ & $1.34 \pm 0.28$ & $106 \pm 9$ & $6869 \pm 211$ \\
\hline Plan_75\% & $95.69 \% \pm 0.38 \%$ & $1.15 \pm 0.09$ & $1.35 \pm 0.33$ & $113 \pm 15$ & $6834 \pm 158$ \\
\hline Plan_80\% & $95.84 \% \pm 0.37 \%$ & $1.16 \pm 0.07$ & $1.33 \pm 0.32$ & $109 \pm 12$ & $6738 \pm 167$ \\
\hline
\end{tabular}

*The value of machine hop were in single fraction radiosurgery.
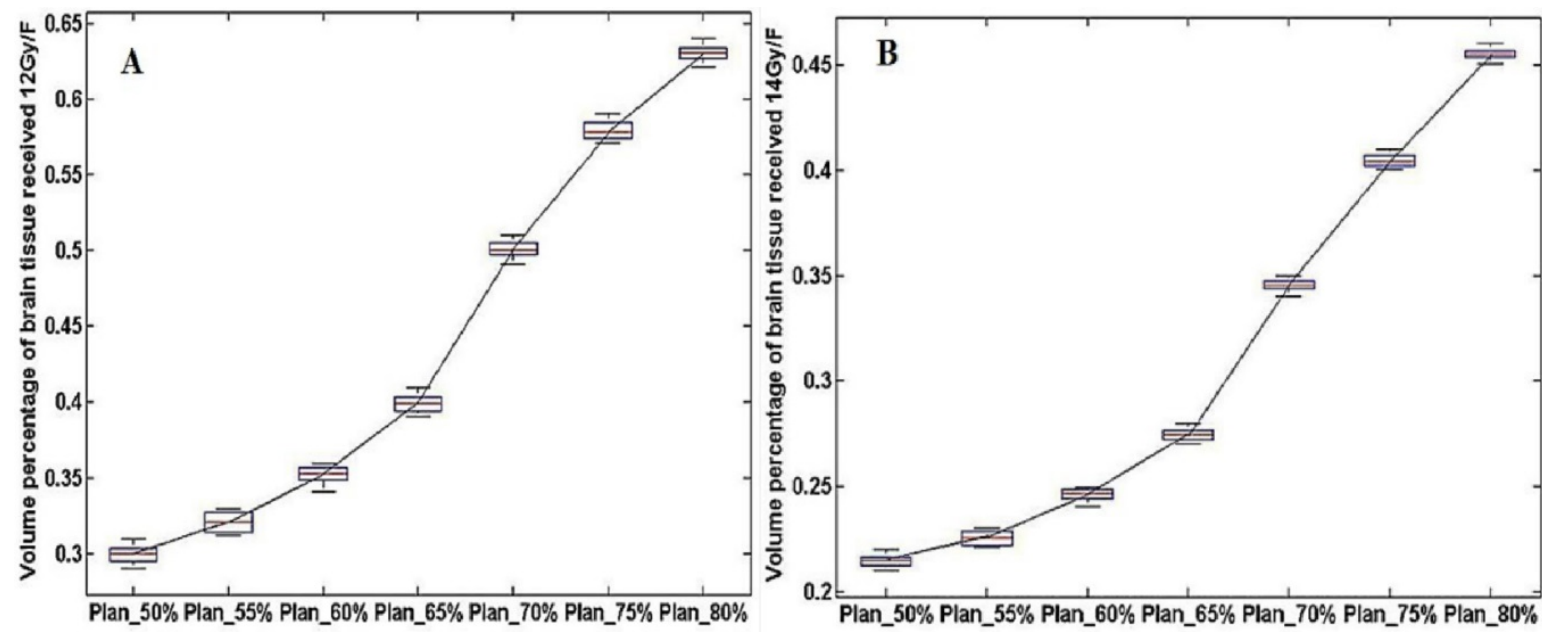

Figure 3. The dosimetry distribution of the whole brain tissue in different plans for the same patients. (A) and (B) were the volume of healthy brain tissue received $12 \mathrm{~Gy} / 14 \mathrm{~Gy}(\mathrm{~V} 12 \mathrm{~Gy} / \mathrm{V} 14 \mathrm{~Gy})$ in a single fraction.

The CI and HI of Plan_50\% and Plan_55\% were significantly different from other plans. That was because it required a bigger collimator to improve dosimetry distribution of the PTV under the same other condition. While it could also lead to uneven dosimetry distribution in the PTV area, Plan_50\% had very hot spots which was not only a spot, but could be a discrete tumor volume. And the machine hop of Plan_50\% and Plan_55\% were higher than other plans. While the difference of total beam counts between plans was not significant. Therefore, Plan_ $50 \%$ and Plan_55\% had the longer treatment time.

The statistical results of V12Gy/V14Gy were shown in Figure 3. It could be observed that V12Gy/ V14Gy value had a marked decline in Plan_65\%. This finding provides more evidence for the theory that
CK SRT plans with $65 \%$ isodose as prescribed dose curve could protect healthy brain tissue better while satisfying the need for clinical treatment.

SRT is used extensively to treat the brain metastases patients who are not clinically suitable for surgery or experience postoperative recurrence [15]. Compared with traditional radiotherapy, SBRT exhibits higher local control rates, effectively reduces intracranial exposure doses, minimizes nerve function injury in patients, and better protects normal brain tissue. CK SRT can achieve high treatment accuracy given its real-time skull matching and tracking function [16,17]. However, due to the single high exposure dose caused by the hypofractionated radiotherapy, it is very easy to cause radiation-induced brain injury. 
Three-fraction (tumors $>10 \mathrm{~cm}^{3}$ ) and five-fraction (large tumors $>30 \mathrm{~cm}^{3}$ ) CK radiotherapy for brain metastases recommended requiring tight control a single dose equivalent of 14 Gy (V14 Gy) to avoid radiation necrosis in patients with metastases [18]. More strict conclusions, the irradiated volume of healthy brain tissue with a single dose equivalent of more than 12 Gy (V12 Gy) was a predictor of brain radio necrosis. The 12 Gy volume of the brainstem is recommended to be decreased to as low as possible in single fraction radiosurgery to reduce the occurrence of any adverse radiation imaging effects on the brainstem and to avoid new neurological deficits [19]. During the CK SRT planning, using different prescription isodose would affect dosimetry distribution in the healthy brain tissue of brain metastases patients. At present, the isodose between $50 \%$ and $80 \%$ will be selected as the prescription dose and there is no uniform standard for it $[20,21]$. In this study, the adoption of the method in which using lower prescription isodose during the development of CK SRT plans could effectively reduce the exposure dose of healthy brain tissue around the PTV and the OAR. However, with utilization of lower normalized isodose as the prescription dose curve, a higher volumetric global maximum was observed. This approach may decrease uniformity of dose distribution and cause necrosis in the PTV volume.

\section{Conclusion}

In this paper, different CK SRT plans for the same patients with brain metastases were designed with different prescription dose line. Although, selecting lower isodose as prescription dose line can increase high dose in tumors and reduce dose delivered to the healthy brain tissues and OAR. But the Plan_50\% and Plan_55\% often needed bigger collimator and had longer treatment time, and higher CI and HI. While Plan_50\% and Plan_55\% had very hot spots which was not only a spot, but could be a discrete tumor volume. Therefore, it is best to choose $60-65 \%$ isodose line as the prescription dose line covered more than $95 \%$ volume of the PTV during CK SRT planning. That could satisfy the need of clinical treatment, while reducing the dosimetry distribution of healthy brain tissue and OAR. These guidelines offer a good protective effect for patients and yield a certain clinical reference value. However, this study is still based on the analysis of the CK SRT planning. Further follow-up of patients who undergo treatment, and statistical analysis of radiation-induced brain necrosis in different clinical design plans should be conducted to provide more powerful evidence for this study and improve the therapeutic effect of patients with brain metastases by CK SRT treatment.

\section{Acknowledgments}

This work was supported by Postdoctoral Research Foundation of China (CN) (No.2019M6610 39) and Research Project of Tianjin Medical University Cancer Institute and Hospital (No.B1715).

\section{Competing Interests}

The authors have declared that no competing interest exists.

\section{References}

1. Higuchi $Y$, Yamamoto M, Serizawa $T$, et al. Modern management for brain metastasis patients using stereotactic radiosurgery: literature review and the authors' gamma knife treatment experiences. Cancer Manag Res. 2018; 10: 1889-1899.

2. Amparo Wolf, Svetlana Kvint, Abraham Chachoua, et al. Toward the complete control of brain metastases using surveillance screening and stereotactic radiosurgery. J Neurosurg. 2017; 128(1): 1-9.

3. Muhammet HB, Kostek O, Erdogan B, et al. Targeted therapy with anaplastic lymphoma kinase inhibitors in non-small cell lung cancer even with brain metastasis. J Buon. 2017; 22(3): 586-591.

4. Nieder C, Andratschke NH, Spanne O, et al. Does overall treatment time impact on survival after whole-brain radiotherapy for brain metastases?. Clin Transl Oncol. 2011; 13(12): 885-888.

5. Young KH, Siddiqui F, Tanyi JA, et al. A retrospective review of sbrt for larger brain metastases or post-resection cavities: preliminary evidence from the knight cancer institute. J Med Imag Radiat On. 2014; 3(3): 253-258.

6. Young K, Siddiqui F, Tanyi J, et al. A retrospective review of sbrt for larger brain metastases or postresection cavities. Int J Radiat Oncol. 2012; 84(3): S295.

7. Park SH, Hwang SK, Kang DH, et al. The retrogasserian zone versus dorsal root entry zone: comparison of two targeting techniques of gamma knife radiosurgery for trigeminal neuralgia. Acta Neurochir. 2010; 152(7):1165-1170.

8. Sio TT, Curran B, Jang S, et al. The Impact of CyberKnife's Prescription Isodose Levels on Intracranial Target Planning. Int J Radiat Oncol. 2011; 81(2):S871-S871.

9. Uno T, Isobe K, Ueno N, et al. Fractionated Stereotactic Radiotherapy as a Boost Treatment for Tumors in the Head and Neck Region. J Radiat Res. 2010; 51(4):449-454.

10. Zindler JD, Bruynzeel AME, Eekers DBP, et al. Whole brain radiotherapy versus stereotactic radiosurgery for 4-10 brain metastases: a phase III randomised multicentre trial. BMC Cancer. 2017; 17(1):500.

11. Zhuang $\mathrm{H}$, Zheng $\mathrm{Y}$, Wang J, et al. Analysis of risk and predictors of brain radiation necrosis after radiosurgery. Oncotarget. 2015; 7(7): 7773-7779.

12. Jang J, Kang YN, Choi BO, et al. Evaluation of the Accuracy of the CyberKnife. Berlin, German: Springer Berlin Heidelberg; 2007.

13. $\mathrm{Xu} \mathrm{Q}$, Fan J, Grimm J, et al. The dosimetric impact of the prescription isodose line (IDL) on the quality of robotic stereotactic radiosurgery (SRS) plans. Med Phys. 2017; 44(12):6159-6165.

14. Lomax NJ and Scheib SG. Quantifying the degree of conformity in radiosurgery treatment planning. Int J Radiat Oncol. 2003; 55(5):1409-1419.

15. Dutta D, Subramanian SB, Murli V, et al. Dosimetric comparison of Linac-based (BrainLAB $®$ ) and robotic radiosurgery (CyberKnife $($ ) stereotactic system plans for acoustic schwannoma. J Neuro-Oncol. 2012; 106(3): 637-642

16. Fink J, Born D, Chamberlain M C. Radiation Necrosis: Relevance with Respect to Treatment of Primary and Secondary Brain Tumors. Curr Neurol Neurosci. 2012; 12(3): 276-285.

17. Kataria T, Narang K, Gupta D, et al. Analysis of intrafraction motion in CyberKnife-based stereotaxy using mask based immobilization and 6D-skull tracking. J Radiosurg SBRT. 2016; 4(3):203-212.

18. Okamoto H, Hamada M, Sakamoto E, et al. Log-file analysis of accuracy of beam localization for brain tumor treatment by CyberKnife. Pract Radiat Oncol. 2016; 6(6):e361-e367.

19. Murovic J, Ding V, Han SS, et al. Impact of cyberknife radiosurgery on overall survival and various parameters of patients with 1-3 versus $\geq 4$ brain metastases. Cureus. 2017; 9(10):e1798.

20. Inoue HK, Sato H, Seto KI, et al. Five-fraction CyberKnife radiotherapy for large brain metastases in critical areas: impact on the surrounding 
brain volumes circumscribed with a single dose equivalent of 14 Gy (V14) to avoid radiation necrosis. J Radiat Res. 2014; 55(2): 334-342.

21. Wiggenraad R, Kanter VD, Kal HB, et al. Dose-effect relation in stereotactic radiotherapy for brain metastases. A systematic review. Radiother Oncol. 2011; 98(3):292-297

22. Motta M, del Vecchio A, Attuati L, et al. Gamma knife radiosurgery for treatment of cerebral metastases from non-small-cell lung cancer. Int J Radiat Oncol. 2011; 81(4):e463-e468. 